\title{
Sistemas de Información de Marketing: una visión integradora
}

\author{
Marketing Information Systems: An integrative view
}

\section{Jesús Manuel López-Bonilla}

Universidad de Sevilla, Facultad de Ciencias Económicas y Empresariales, Departamento de Administración de Empresas y Marketing, Ramón y Cajal no 1 - 41018, Sevilla - España, lopezbon@us.es

\section{Luis Miguel López-Bonilla}

Universidad de Sevilla, Facultad de Ciencias Económicas y Empresariales, Departamento de Administración de Empresas y Marketing, Sevilla - España, luismi@us.es

\section{Jesús C. Peña-Vinces}

Universidad de Sevilla, Facultad de Ciencias Económicas y Empresariales, Departamento de Administración de Empresas y Marketing, Sevilla - España, jesuspvinces@us.es

\section{Resumen}

La información es un recurso imprescindible en la toma de decisiones de las organizaciones. Por ello, las organizaciones recurren a sistemas de información que les provean de este recurso básico. Un sistema de información es un medio por el que fluyen datos en una organización, desde un trabajador o departamento hasta otros trabajadores o departamentos. Esto puede ocurrir desde la comunicación interna de la organización hasta los sistemas informatizados que generan informes periódicos para distintos usuarios.

En el presente trabajo, nos centramos en los sistemas de información desde la perspectiva de marketing. Se ha llevado a cabo un enfoque de estudio cualitativo, realizando una revisión de los modelos más relevantes presentados históricamente en la literatura académica respecto a los sistemas de información de marketing. Los sistemas más comunes son el Sistema de Información de Marketing (SIM) y el Sistema de Apoyo a las Decisiones de Marketing (SADM). Hemos observado que existe una cierta confusión en la literatura respecto a estos sistemas de información. Así, hemos tratado de aportar una visión integradora de los distintos puntos de vista existentes, proponiendo un sistema híbrido y global.

Palabras claves: Sistema de información, sistema de información de marketing, SIM, sistema de apoyo a las decisiones de marketing, SADM.

\begin{abstract}
Information is a basic resource for the decision making. For this, the organizations use information systems which give this basic resource. An information system is a means through which data flow inside a company, from a worker or department to other workers or departments. This may occur everywhere, from the organizations's internal communication to computerized systems that generate periodical reports for varied users.

In the present work, we center on the information systems from a marketing perspective. The methodology of this paper is based on a qualitative research approach. This article reviews relevant marketing information system models presented historically in the academic literature. The information systems most commonly examined are the Marketing Information System (MIS) and the Marketing Decision Support System (MDSS). We have observed that there is some confusion in the literature regarding these information systems. Hence, we have aimed to give an integrative viewpoint and we have proposed a hybrid and global system.
\end{abstract}

Keywords: Information system, marketing information system, MIS, marketing decision support system, MDSS.

\section{Introducción}

Es sobradamente conocido que la información es un recurso imprescindible en la toma de decisiones de las organizaciones. La experiencia de los responsables de las organizaciones se debe complementar con la información necesaria para tratar de reducir riesgos y tomar las decisiones más adecuadas. Por ello, las organizaciones recurren a sistemas de información que les provean de este recurso básico. Además, como indican Pérez-López y Junquera (2013), las empresas se enfrentan a un entorno competitivo caracterizado por la globalización de los mercados, la complejidad creciente de los problemas empresariales y la aceleración del fenómeno de cambio.

Esteban (1996) define el sistema de información como un medio a través del cual fluyen los datos desde una persona o departamento hacia otros y puede ser desde la comunicación interna de la organización hasta sistemas informatizados que generen informes periódicos por varios usuarios. Los sistemas de información en la empresa fueron concebidos en la década de los años sesenta del siglo XX como un instrumento al servicio de la función meramente operativa, aunque las nuevas tendencias y los adelantos tecnológicos los han encauzado hacia las funciones tácticas y estratégicas, sin perturbar el rendimiento de su primera aplicación (García de Madariaga, 1994). Como advierten Romeiro y Garmendia (2007), los sistemas de información se han centrado en los niveles operativos, es decir, donde se concentra el mayor flujo de información contable, financiera, de ventas y logística. Sin embargo, la dirección de marketing debe ofrecer también una perspectiva estratégica que respalde el componente operativo de la empresa.

García de Madariaga (1994) subraya que, frente a la tendencia de instaurar sistemas de información totales o monolíticos para satisfacer las necesidades de una organización en todos sus niveles, prevalece el concepto de descentralización de estos sistemas por áreas funcionales, cada uno con su propia visión y finalidad. En este sentido, la función de marketing exige la construcción de unos 
sistemas que le permitan analizar la evolución del entorno, identificar la competencia, estudiar el atractivo del mercado y las expectativas y deseos de los consumidores.

En el presente trabajo, nos centramos en los sistemas de información de las organizaciones desde la perspectiva de marketing. La metodología de estudio está basada en un enfoque cualitativo que ha consistido en la revisión crítica de los sistemas de información de marketing. Para ello, se han revisado y discutido los modelos que describen los sistemas de información de marketing en la literatura académica. Esta revisión de la literatura comprende revistas académicas $\mathrm{y}$, especialmente, libros y manuales, debido a que en estos últimos es donde se ha encontrado un mayor material de referencia. Hemos detectado en la revisión de la literatura que existe una cierta confusión respecto a su estructura e identificación, por lo que el objetivo principal de este trabajo es aportar algo de luz al conocimiento de estos sistemas de información, tratando de llegar a una idea más integradora de los distintos puntos de vista existentes. Por tanto, esta revisión crítica de los enfoques teóricos sobre el sistema de información de marketing deriva en una propuesta de un modelo integrador que incluye los cambios producidos en su evolución a lo largo del tiempo. Este modelo de carácter híbrido se presenta de manera clara, lógica e interrelacionada. De este modo, creemos que este trabajo contribuye a la literatura en la medida que trata de comprender la evolución de los sistemas de información de marketing.

\section{Sistema de Información de Marketing}

El Sistema de Información de Marketing (en adelante, SIM) supone un tópico clásico en la investigación académica. Algunos estudios se han centrado sobre el desarrollo del SIM (v.g., Darena, 2007; Lin \& Hong, 2009; MatviychukSoskina, 2009), el uso del SIM (v.g., Li, 1995; Li, Chen \& Roan, 2001a; Li, McLeod \& Rogers, 2001b), las aplicaciones del SIM (v.g., Wöber, 1994; Ritchie \& Ritchie, 2002; Molina, Gómez y Martín-Consuegra, 2010) y las consecuencias de la adopción del SIM (O’Brien, Schoenbachler \& Gordon, 1995; Talvinen \& Saarinen, 1995; Van Bruggen, Smidts \& Wierenga, 1998). En cambio, hay algún otro aspecto poco estudiado todavía, como es la medida de la efectividad del SIM (Gounaris, Panigyrakis \& Chatzipanagiotou, 2007).

Especialmente, la relación del SIM y las tecnologías de la información concentra un gran interés investigador (v.g., Talvinen, 1995; Hess, Rubin \& West, 2004; Leonidou \& Theodosiou, 2004; Kothari, Xiang \& Fesenmaier, 2008). Por ejemplo, las tecnologías de la información (TIC) están siendo cada vez más utilizadas por las empresas para tratar de ganar ventajas competitivas sostenibles. La estrategia TIC envuelve al sistema de información (Jelonek, 2013). El valor de la inversión en sistemas de información y TIC, no considerándolo como estratégico, reduce su capacidad de obtener beneficios a partir de su uso (Azevedo, Romao y Rebelo, 2014). El uso de las TIC para apoyar la función de marketing no ha sido diferente. Además, como Toivonen (1999) sugiere, el sistema de información de marketing está físicamente incorporado en las tecnologías de la información, es decir, el hardware y el software se usan para construir este sistema. Las TIC pueden proporcionar oportunidades para obtener ventajas competitivas o ser una necesidad competitiva. Uno de los primeros beneficios que una empresa obtiene del sistema de información de marketing basado en TIC es la mejora del sistema de informes (Gounaris, Panigyrakis \& Chatzipanagiotou, 2007). Un segundo beneficio importante es la capacidad de vigilar el entorno de la empresa con mayor efectividad, específicamente en lo que respecta a las relaciones con los clientes y, de este modo, asistir a los gestores y agentes de venta en la consecución de sus objetivos (Speier \& Venkatesh, 2002). La implantación del sistema de información de marketing basado en TIC puede cambiar potencialmente el papel de la función de marketing de manera radical y ayudar a aumentar el grado de orientación al cliente que posee la empresa (Nakata \& Zhu, 2006). Además, la evolución de las tecnologías Web 2.0 ha traído oportunidades de negocio significativas para las organizaciones y para los consumidores. En las redes sociales online, las personas reciben y proporcionan una gran corriente de información. De esta manera, este entorno ofrece una oportunidad excelente para el desarrollo de los sistemas de información de marketing.

En un estudio pionero de Cox y Good (1967), el Sistema de Información de Marketing se definía como el conjunto de procedimientos y métodos para la recopilación, el análisis y la presentación de información para utilizarla en la toma de decisiones de marketing. Marshall y LaMotte (1992) establecen que el SIM es un sistema integral, flexible, formal y evolutivo para tener un flujo organizado de información pertinente para orientar la toma de decisiones de marketing. Siguiendo a Marshall (1996), esta definición se basa en tres características principales, como comentamos a continuación. En primer lugar, el SIM debe concebirse como integral y flexible, ya que las actividades de marketing de una compañía están relacionadas entre sí y deben adaptarse a entornos cambiantes. En segundo término, el SIM debe ser formal y evolutivo, es decir, se debe diseñar a conciencia al señalar metas organizativas específicas, para que satisfaga las necesidades de los gerentes de marketing durante un amplio período de tiempo. Por último, el SIM debe brindar un flujo organizado de información pertinente para orientar la toma de decisiones de marketing.

El SIM está basado en un proceso sistemático de información con alto valor estratégico, abarcando redes sociales de clientes, profesionales y otros agentes (Morales \& Hernández, 2011). Como López-Bonilla y López-Bonilla (2012; 2014) indican, Phillip Kotler y sus colaboradores han refinado y divulgado el concepto del SIM, definiéndolo como una estructura estable de interacción, integrada por un conjunto de personas, instrumentos y procedimientos dedicados a la recogida, clasificación, análisis, valoración y distribución de la información para el uso de los responsables de marketing (Kotler \& Armstrong, 1991). Dicho autor y sus colaboradores han propuesto un modelo estructural del SIM ampliamente conocido, basado en cuatro componentes o subsistemas, que se detallan seguidamente (ver figura 1). 
Figura 1 - Sistema de Información de Marketing (SIM)

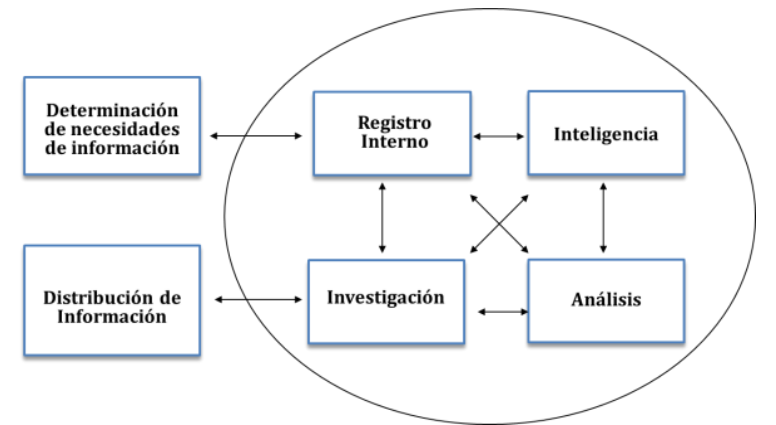

Fuente: adaptado de Kotler y Armstrong (1991).

Subsistema de registro interno: se dedica a gestionar los datos propios de la organización que se generan a partir de su actividad diaria. Como indican Grande y Abascal (2006), recoge los flujos de información de las organizaciones con su microentorno. Por ejemplo, los datos se obtienen a partir de los registros de ventas, las reclamaciones de los clientes, los informes de los vendedores o los estados de cuentas.

Subsistema de inteligencia: se ocupa de gestionar los datos que provienen del entorno de la organización en su relación de intercambio permanente. Es decir, se recoge información sobre los aspectos económicos, sociales, políticos, legales, medioambientales, físicos o tecnológicos. Entre las fuentes de datos se encuentran los propios organismos e instituciones, públicos y privados, como las asociaciones profesionales, las administraciones públicas, las cámaras de comercio o las universidades. Asimismo, los datos se pueden extraer también de las publicaciones técnico-profesionales, revistas especializadas y prensa. Por último, una fuente de información externa muy valiosa está relacionada con los contactos permanentes del personal de la organización con los clientes, distribuidores y proveedores.

Subsistema de investigación: se centra en el estudio de hechos concretos que son necesarios en un momento del tiempo determinado para resolver un problema comercial específico. Por ejemplo, cuando una empresa quiere conocer la aceptación de un nuevo producto por parte de los consumidores potenciales.

Subsistema de análisis: se encarga de realizar las pruebas y valoraciones cualitativas y cuantitativas que son oportunas para orientar a los responsables de marketing en su toma de decisiones. Se compone de un banco de modelos y un conjunto de herramientas estadísticas.

De manera genérica, los tres primeros subsistemas se dedican a la recopilación de datos, mientras que el cuarto subsistema se centra en su evaluación y transformación en información. Como señalan Díez y Landa (2002), la investigación comercial es la parte sustancial del SIM. La investigación comercial se convierte en el elemento central del SIM porque su razón de existencia está supeditada a las carencias de los subsistemas de registro interno y de inteligencia. Es decir, si estos dos últimos subsistemas no pueden proporcionar la información necesaria para tomar ciertas decisiones, entonces es cuando se requiere la participación del subsistema de investigación para recabar dicha información. Y este hecho suele suceder con frecuencia.

\section{Sistema de Apoyo a las Decisiones de Marketing}

Aaker, Kumar y Day (2000) subrayan que el SIM se ocupa de administrar el flujo de datos proveniente de muchos proyectos, estudios y fuentes secundarias hacia los posibles gerentes que lo van a utilizar. Para ello, estos autores indican que se necesita el uso de bases de datos para organizarlos y almacenarlos y, además, se requiere de un sistema de apoyo a las decisiones para recuperar los datos y transformarlos en información, así como distribuir dicha información a los usuarios. Esto último se conoce con el término de Sistema de Apoyo a las Decisiones de Marketing (abreviadamente, SADM).

Pero los primeros conceptos de los sistemas de apoyo a las decisiones gerenciales surgen a comienzos de los años setenta, aunque su desarrollo haya sido posterior, dado que están supeditados a las capacidades informáticas. Así, ScottMorton (1971) emplea el término de sistema de gestión de decisiones para definirlo como un sistema interactivo basado en ordenador, que ayuda a la toma de decisiones en el uso de datos y modelos para resolver problemas no estructurados.

Little (1979) describe el SADM como la colección coordinada de datos, sistemas, herramientas y técnicas, con programas y ordenadores de apoyo, que permite a una organización obtener e interpretar la información relevante sobre su actividad y su entorno y hacer de ella la base de sus actividades de marketing. Dillon, Madden y Firtle (1994) establecen una clara diferencia a través de la orientación humanística que posee el SADM, considerando que la solución de los problemas comerciales se halla en la propia dirección de la organización y no tanto en la tecnología utilizada. En un mismo sentido, Trespalacios, Vázques y Bello (2005) sostienen que el SADM asume los cambios sustanciales producidos por las nuevas tecnologías de la información que hacen posible una ayuda mucho más directa y adaptada al decisor.

Luque (1997) señala que ambos sistemas de información proporcionan apoyo a las decisiones, pero el SADM es un sistema que dispone de una modelización más desarrollada que el SIM. En este sentido, como apunta Malhotra (1997), el SADM se ha desarrollado para superar las limitaciones del SIM. Este autor indica que ambos sistemas combinan el uso de modelos o técnicas analíticas con las funciones de acceso y recuperación tradicionales del SIM; ambos son fáciles de usar de modo interactivo y pueden adaptarse tanto a los cambios del entorno como al enfoque de toma de decisiones que siga el usuario. Pero, siguiendo a Gupta y Kohli (1990), se puede subrayar una serie de diferencias entre ellos, que incluimos en la tabla 1. 


\section{Tabla 1 - Diferencias entre SIM y SADM}

\begin{tabular}{|c|c|}
\hline SIM & SADM \\
\hline Problemas estructurados & Problemas no estructurados \\
\hline Uso de informes & Uso de modelos \\
\hline Estructura rígida & Interacción amigable con el usuario \\
\hline Exposición restringida de la información & Adaptabilidad \\
\hline $\begin{array}{l}\text { - Se puede mejorar la toma de decisiones } \\
\text { depurando los datos primarios }\end{array}$ & $\begin{array}{l}\text { - Se puede mejorar la toma de decisiones } \\
\text { utilizando el análisis prospectivo }\end{array}$ \\
\hline
\end{tabular}

Fuente: adaptado de Malhotra (1997).

Hair, Bush y Ortinau (2003) plantean un esquema gráfico de la estructura del SADM (ver figura 2) y distinguen una serie de características singulares del SADM frente al SIM, como se enumeran a continuación: (1) está diseñado para atender problemas de investigación demandados por el personal de marketing; (2) ofrece información destinada a facilitar una decisión concreta; (3) su principal objetivo es evaluar varias soluciones para los problemas de marketing e identificar la mejor acción; (4) está orientado a resolver problemas específicos de marketing; y (5) pone énfasis en el almacenaje y clasificación de la información.

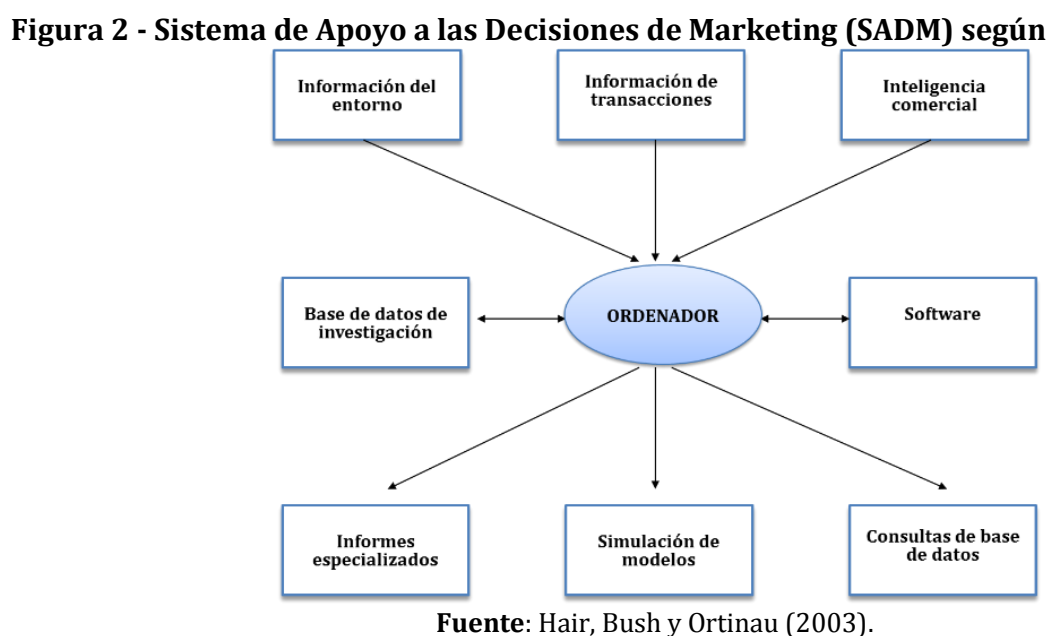

\section{Propuesta de un sistema integrador}

Hemos comprobado en la revisión de la literatura que el Sistema de Información de Marketing se confunde habitualmente con el Sistema de Apoyo a las Decisiones de Marketing. Las definiciones de ambos sistemas parece que no son lo suficientemente aclaratorias y excluyentes. Quizás esto sea debido a que el SIM y el SADM se han tratado en la literatura como dos sistemas distintos pero también se han concebido de manera conjunta.

Como García de Madariaga (1994) aprecia, el subsistema de análisis ha evolucionado, como consecuencia de la inestabilidad del entorno, hasta erigirse como un sistema autónomo diseñado de forma personalizada en torno a los directivos, en función de las necesidades de información y análisis previos a su toma de decisiones. Esto ha dado lugar a la construcción del SADM. En este sentido, Gómez, Mollá y Gil (2000) afirman que estos sistemas enfatizan atributos como la flexibilidad y un enfoque ad hoc al abordar el objetivo de proporcionar información relevante para la decisión, por lo que la filosofía del SADM es superior a la del SIM en tanto que incluye a éstos y amplía su funcionalidad.

Por otro lado, la estructura del SADM presenta una gran divergencia en cuanto a su número y tipos de componentes. Así, por ejemplo, Zikmund (1997) los resume en dos componentes: base de datos y software; Dillon, Madden y Firtle (1994) sugieren tres elementos esenciales: base de datos, interfaz de usuario y banco analítico y de diseño. Churchill (1995) destaca también tres componentes, aunque los denomina como sistema de datos, sistema de modelos y sistema de diálogo. Aaker, Kumar y Day (2000) optan por cuatro componentes: base de datos, informes y presentaciones, capacidades de análisis y modelos. Dyer y Forman (1991) sugieren cinco componentes: banco estadístico, banco de modelos, banco de datos, reglas de optimización e interfaz de usuario. Marshall (1996) se decanta también por cinco elementos, aunque distintos a los anteriores, como son: sistema de informes y consultas, modelos analíticos, sistema de apoyo ejecutivo, sistema de apoyo a las decisiones grupales y sistema de inteligencia artificial. Por último, Malhotra (1997) indica que el SADM incluye seis aspectos, tales como hardware, software, red de comunicación, base de datos, modelos y usuarios.

Esta división de opiniones puede explicarse a través de las palabras de McDaniel y Gates (2005), que apuntan que el sistema ideal no se ha realizado en la práctica. No obstante, el componente fundamental que aparece en todas las estructuras ideadas es el de la base de datos. En cambio, el componente software parece que distorsiona la estructura del SADM porque se extiende por todos sus componentes, actuando más bien como la savia del sistema. El SADM debe estar construido en función de elementos razonablemente distintos entre sí y por tanto la mejor opción teórica parece ser la aportada por Aaker, Kumar y Day (2000). A continuación, detallamos la clasificación en la que estos autores se han basado para identificar los componentes del SADM (ver figura 3). Curiosamente, esta estructura del SADM es una adaptación casi exacta del SIM propuesto por Montgomery y Urban (1970), basado en el uso del ordenador. 
Figura 3 - Sistema de Apoyo a las Decisiones de Marketing (SADM) según Aaker, Kumar y Day (2000)

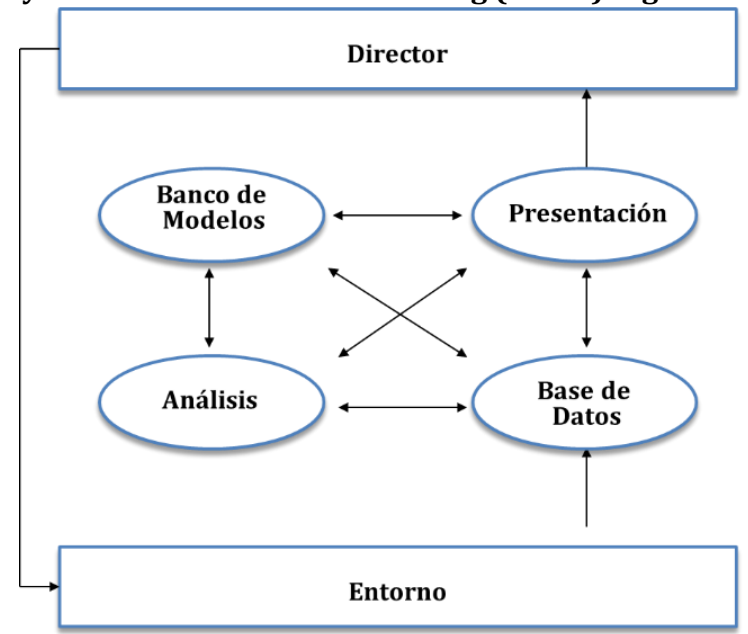

Fuente: Aaker, Kumar y Day (2000).

- Base de datos. Es un conjunto de datos dispuestos en forma lógica y organizados de manera que un ordenador puede almacenarlos y procesarlos (Zikmund, 1997). Se sustenta en los registros de datos de todo tipo de fuentes internas y externas a la organización.

- Capacidades de análisis. Se trata de disponer de los medios y las habilidades suficientes para poder realizar cálculos y establecer relaciones entre los datos. Se sirve de las diversas aplicaciones estadísticas existentes o incluso de las propias aplicaciones desarrolladas por la organización.

- Banco de modelos. Constituye una serie de esquemas teóricos que representan una realidad compleja con objeto de facilitar su comprensión. Se basa en la simulación de las relaciones entre las distintas variables de marketing.

- Capacidades de diseño o presentación. Se refiere a las diversas opciones de formato de los resultados obtenidos por el usuario. Se incluye la presentación de cualquier tipo de gráficos, tablas o documentos que favorezca una rápida y clara idea de la consulta planteada, que se puede considerar, en términos generales, como un informe.

Como se ha podido apreciar, las estructuras del SIM y del SADM son diferentes. El SIM aparenta una mayor inclinación hacia la determinación y búsqueda de las fuentes de información apropiadas, mientras que el SADM hace un mayor énfasis hacia el tratamiento y gestión de esas fuentes de forma eficiente, aprovechando los recursos tecnológicos, y de forma eficaz, facilitando su conexión con el usuario. Pero estos sistemas son más complementarios que excluyentes. Es decir, hay que entenderlos en una relación de continuidad más que de solapamiento. Por ello, es posible establecer una configuración híbrida entre ambos. Algunos autores ya lo habían propuesto, pero se han limitado a sustituir el subsistema de análisis del SIM por el Sistema de Apoyo a las Decisiones de Marketing. Creemos conveniente representar una estructura nueva a partir de los dos sistemas evitando un exceso de simplificación. En la figura 4 se representa esta estructura, a la que hemos asignado el término de Sistema Global de Información de Marketing (abreviadamente, SGIM).

Figura 4 . Sistema Global de Información de Marketing (SGIM)

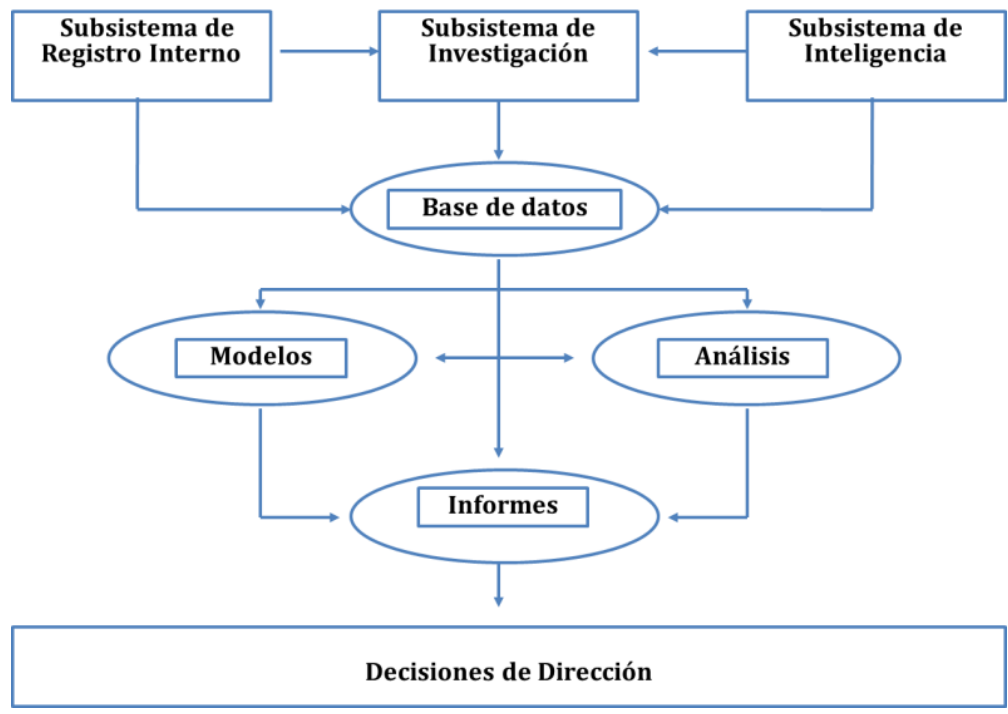

Fuente: elaboración propia. 
En definitiva, el SGIM está compuesto por dos grandes apartados en los que se distingue, por un lado, la obtención de datos internos y externos a la organización a través de los tres subsistemas de registro interno, investigación e inteligencia. Se puede observar la mayor preponderancia del subsistema de investigación respecto a los subsistemas de registro interno e inteligencia, en consonancia con los que indican Díez y Landa (2002), que se sitúa como eje central en la primera parte de la representación gráfica. Y, por otro lado, hay un segundo apartado centrado en la transformación óptima de los datos en información veraz, relevante y oportuna para la gerencia. Como nexo de unión entre estos dos apartados del sistema se encuentra la gestión de la base de datos.

\section{Conclusiones}

La información supone un recurso esencial para las organizaciones en el desarrollo de su actividad cotidiana en un mundo cada vez más complejo y dinámico. El uso de sistemas de información supone una herramienta importante para facilitar el proceso de decisiones que debe asumir la organización y que va a procurarle, posiblemente, un menor riesgo de fracaso y un aumento de su capacidad de gestión.

En el presente trabajo hemos tratado de comprender los sistemas de información en el área de marketing y nos hemos centrado especialmente en analizar su estructura. Hemos observado que hay divergencias conceptuales respecto a estos sistemas de información (v.gr., Kotler \& Armstrong, 1991; Malhotra, 1997; Aaker, Kumar \& Day, 2000; Hair, Bus \& Ortinau, 2003). Pero estas divergencias también están basadas en la evolución en el tiempo y en la adaptación a las nuevas tecnologías de la información. Para Kotler y Armstrong (1991), el sistema de información de marketing es un modelo clásico para la educación de marketing, como el modelo famoso de las cuatro p. Sin embargo, como indica Toivonen (1999), el sistema de información de marketing está incluido físicamente en las tecnologías de la información. Por lo tanto, las TIC han cambiado la estructura del SIM con idea de proporcionar una ventaja competitiva sostenible. De acuerdo con Nakata y Zhu (2006), este cambio puede ayudar a incrementar la orientación de mercado de las empresas.

Los dos conceptos relativos a los sistemas de información en el área de marketing que son más habituales actualmente son el Sistema de Información de Marketing (SIM) y el Sistema de Apoyo a las Decisiones de Marketing (SADM). Centrándonos en la estructura del sistema de información de marketing, se ha observado en la revisión de la literatura una cierta confusión. En este sentido, se ha comprobado que es frecuente que el SIM se confunda con el SADM. Por una parte, parece claro que el SIM esté basado en cuatro componentes o subsistemas (Kotler \& Armstrong, 1991). Sin embargo, el SADM muestra diferentes estructuras que se basan en dos o más componentes (v.gr., Churchill, 1995; Marshall, 1996; Malhotra, 1997; Zikmund, 1997; Aaker, Kumar \& Day, 2000). Este segundo sistema ha evolucionado a partir del primero, pero no son sustitutivos. Sin embargo, creemos que no se ha dado todavía una visión integradora de ambos conceptos, sino que se han definido de una manera aislada y algo confusa. Por ello, hemos tratado de proponer un marco teórico en el que basarnos para establecer una estructura común de estos sistemas dando lugar a lo que hemos denominado como Sistema Global de Información de Marketing (SGIM). Este sistema híbrido se compone de dos grandes bloques o funciones, basados tanto en la recogida de datos internos y externos a la organización como en la transformación óptima de estos datos para que la gerencia disponga de información pertinente para la toma de decisiones.

Como el mercado global es cada vez más competitivo y complejo, es necesario procesar muchos más datos para la toma de decisiones empresariales. Para afrontar esta competencia e incertidumbre crecientes, las empresas requieren alcanzar ventajas de las TIC y de los sistemas de información (Nasir, 2005). Hoy en día, ser competitivos en la sociedad actual significa tener un enfoque tecnológico que aporte respuestas al consumidor (Ramos, 2010). En este sentido, los sistemas de información de marketing basados en TIC pueden ayudar en esta dirección (Talvinen, 1995). El SGIM obtiene ventajas de las TIC y de los sistemas de información y esto proporciona un mayor desarrollo que el SIM o el SADM. Así, el SGIM es un modelo ampliado, pero es también un modelo más preciso y comprensivo que los otros dos modelos citados anteriormente.

Como ya se ha dicho, la literatura académica presenta una cierta confusión entre los modelos SIM y SADM, pero la combinación de estos dos modelos tradicionales puede superar estas limitaciones conceptuales. Precisamente, el propósito del modelo SGIM es integrar ambos sistemas dentro de una estructura holística y significativa. El SGIM es un modelo interdisciplinar, que implica a las áreas de marketing, sistemas de experto, bases de datos y estadística e investigación operativa. En general, el SGIM puede ser comprendido mejor a través de su descomposición entre la investigación de marketing, la inteligencia de marketing y la gestión de base de datos. Como Lin y Hong (2009) apuntan, la investigación de marketing y la inteligencia de marketing permiten la recogida y la presentación de información a los gestores con el propósito de tomar decisiones mejores y más rápidas. En cambio, la gestión de base de datos representa la transformación óptima de los datos en información pertinente. Esto último significa el componente esencial del SGIM dado que es un vínculo entre los dos modelos tradicionales.

\section{References}

Aaker, D. A., Kumar, V., \& Day, G. S. (2000). Marketing research. New York: John Wiley \& Sons Inc.

Azevedo, P.S., Romao, M., \& Rebelo, E. (2014). Success factors for using ERP Enterprise Resourse Planning) systems to improve competitiveness in the hospitality industry. Tourism \& Management Studies, 10(Special Issue), 165-168.

Churchill, G. A. (1995). Marketing research. Methodological foundations. Hillsdale, IL: Dryden Press.

Cox, D. F., \& Good, E. (1967). How to build a marketing information system. Harvard Business Review, 45(3), 145.

Darena, F. (2007). Global architecture of marketing information systems. Agricultural Economics, 53(9), 432-440.

Díez, E. C., \& Landa, J. (2002). Marketing. Investigación comercial. Madrid: Ediciones Pirámide. 
Dillon, W. R., Madden, T. J., \& Firtle, N. H. (1994). Marketing research in a marketing environment. Homewood, IL: Irwin.

Dyer, R. F., \& Forman, E. H. (1991). An analytic approach to marketing decisions. New York: Prentice-Hall.

Esteban, A. (1996). Principios de marketing. Madrid: ESIC Editorial.

García de Madariaga, J. (1994). Análisis y evolución de los sistemas de información de marketing. ESIC-Market, 83, 51-62.

Grande, I., \& Abascal, E. (2006). Fundamentos y técnicas de investigación comercial. Madrid: ESIC Editorial, 2006.

Gómez, M. A., Mollá, A., \& Gil, I. (2000). El papel de la investigación comercial en el marketing. En J. Martínez, F. Martín, E. Martínez, L. A. Sanz y C. Vacchiano (Eds), La investigación en marketing (pp. 3354). Barcelona: AEDEMO.

Gupta, S., \& Kohli, R. A. (1990). Knowledge-based system for advertising design. Marketing Science, 9(3), 230-246.

Gounaris, S. P., Panigyrakis, G. G., \& Chatzipanagiotou, K. C. (2007). Measuring the effectiveness of marketing information systems: An empirically validated instrument. Marketing Intelligence \& Planning, 25(6), 612-631.

Hair, J. F., Bush, R. P., \& Ortinau, D. J. (2003). Marketing research: Within a changing information environment. New York: McGraw-Hill.

Hess, R.L., Rubin, R.S., \& West, L.A. (2004). Geographic information systems as a marketing information system technology. Decision Support System, 38(2), 197-212.

Jelonek, D. (2013). Synergistic effect in alignment between business environment scanning strategy and IT strategy in enterprises. Tourism \& Management Studies, 9(1), 116-120.

Kothari, T., Xiang, Z., \& Fesenmaier, D.R. (2008). The impact of a marketing information system: a case study of Smart-Baltimore. Journal of Information Technology Tourism, 10(1), 59-74.

Kotler, P., \& Armstrong, G. (1991). Principles of marketing. Englewood Cliffs, NJ: Prentice-Hall.

Leonidou, L.C., \& Theodosiou, M. (2004). The export marketing information system: an integration of the extant knowledge. Journal of World Business, 39(1), 12-36.

Li , E.Y. (1995). Marketing information systems in the top U.S. companies: A longitudinal analysis. Information and Management, 28(1), 13-31.

Li, E.Y., Chen, H.-G., \& Roan, J. (2001a). Marketing information systems usage in Taiwan's top 1000 companies. Journal of Computer Information Systems, 41(2), 85-94.

Li, E.Y., McLeod, R., \& Rogers, J. C. (2001b). Marketing information systems in Fortune 500 companies: A longitudinal analysis of 1980, 1990, and 2000. Information and Management, 38(5), 307-322.

Lin, C., \& Hong, C. (2009). Development of a marketing information system for supporting sales in a tea-beverage market. Expert Systems with Applications, 36(3), 5393-5401.

Little, J. D. C. (1979). Decision support systems for marketing managers. Journal of Marketing, 43(3), 9-26.

López-Bonilla, J. M., \& López-Bonilla, L. M. (2012). Investigación de mercados turísticos. Madrid: Ediciones Pirámide.

López-Bonilla, J. M., \& López-Bonilla, L. M. (2014). Investigación de mercados: teoría y casos. Madrid: Pearson.

Luque, T. (1997). Investigación de marketing. Fundamentos. Barcelona: Ariel.

Malhotra, N. K. (1997). Marketing research. An applied orientation. Englewood Cliffs, NJ: Prentice-Hall.

Marshall, K. P. (1996). Marketing information systems: Create competitive advantage in the information age. Boston, MA: Boyd \& Fraser Publishing Co.

Marshall, K. P., \& Lamotte, S. W. (1992). Marketing information systems: A marriage of systems analysis and marketing management. Journal of Applied Business Research, 8(3), 61-73.

Matviychuk-Soskina, N.0. (2009). Analysis of contemporary approaches to construction of marketing information systems. Actual Problems of Economics, 8, 266-272.

McDaniel, C., \& Gates, R. (2005). Marketing research. New York: John Wiley \& Sons Inc.
Molina, A., Gómez, M., \& Martín-Consuegra, D. (2010). Tourism marketing information and destination image management. African Journal Business Management, 4(5), 722-728.

Morales, G., \& Hernández, J. M. (2011). Los stakeholders del turismo. Tourism \& Management Studies, Special Issue (I), 894-903.

Montgomery, D. B., \& Urban, G. L. (1970). Marketing decision information systems: An emerging view. Journal of Marketing, 7(2), 227. Nakata, C., \& Zhu, Z. (2006). Information technology and customer orientation: A study of direct, mediated, and interactive linkages. Journal of Marketing Management, 22, 319-54.

Nasir, S. (2005). The development, change, and transformation of management information systems (MIS): A content analysis of articles published in business and marketing journals. International Journal of Information Management, 25, 442-457.

O'Brien, V.T., Schoenbachler, D.D., \& Gordon, G.L. (1995), Marketing information systems for consumer products companies: a management overview. Journal of Consumer Marketing, 12(5), 16-36.

Pérez-López, S., \& Junquera, B. (2013). The relation between IT competency and knowledge management processes and its mediators. Tourism \& Management Studies, 9(1), 109-115.

Ramos, C. (2010). Information systems for tourism management. Tourism \& Management Studies, 6, 107-116.

Ritchie, R. J. B., \& Ritchie, J. R. B. (2002). A framework for an industry supported destination marketing information system. Tourism Management, 23(5), 439-454.

Romeiro, J., \& Garmendia, F. (2007). Sistemas de información de marketing (SIM): más que simples cajas tecnológicas. ESIC-Market, 128, 131-143.

Scott-Morton, M. S. (1971). Management decision systems: Computer-based support for decision making. Cambridge, MA: Harvard University.

Speier, C., \& Venkatesh, V. (2002). The hidden minefields in the adoption of sales force automation technologies. Journal of Marketing, 66(3), 98-111.

Talvinen, J. M. (1995). Information systems in marketing. Identifying opportunities for new applications. European Journal of Marketing, 29(1), 8-26.

Talvinen, J. M., \& Saarinen, T. (1995). MkIS support for the marketing management process: Perceived improvements for marketing management. Marketing Intelligence \& Planning, 13(1), 18-27.

Toivonen, R. (1999). Planning the use of information technology in marketing: The case of Finnish forest industries. Forest Products Journal, 49(10), 25-30.

Trespalacios, J. A., Vázquez, R., \& Bello, L. (2005). Investigación de mercados. Madrid: Thomson.

Van Bruggen, G., Smidts, H., \& Wierenga, B. (1998). Improving decision making by means of a marketing decision support system. Management Science, 44(5), 614-45.

Wöber, K.W. (1994). Tourism marketing information system. Annals of Tourism Research, 21(2), 396-399.

Zikmund, W. G. (1997). Exploring marketing research. New York: Prentice-Hall.

Recibido: 13.04.2014

Aceptado: 14.10.2014 